\title{
A signaling scheme for B-ISDN and IN integration
}

\author{
Claude Rigault \\ Ecole Nationale Supérieure des Télécommunications, Paris, France \\ e-mail rigault@res.enst.fr \\ Tatiana Kováciková \\ University of Zilina, Slovakia \\ e-mailtana@frkis.utc.sk
}

Keywords : B-ISDN signaling, Control plane, Call Model, Session concept

Abstract

This paper proposes an architecture for the control plane of the B-ISDN network. It first explains the requirements for Service / Connection separation and Call / Bearer separation, leading to the Service Session Concept and the Call Concept as separated entities. After explaining the requirements for an Access Session, the Service Precedence principle is introduced. According to this principle, only when the service is activated prior to the call, a universal call model may be designed. Our proposed architecture is then described, showing how the precedence principle is respected. Both, the functional architecture and the physical architecture are described, which lays the ground for defining all the signaling paths required. After a brief description of the characteristics of each of these signaling paths, and of the corresponding signaling protocols, two examples of services are explained.

\section{1) Introduction}

This paper is a contribution to the future B-ISDN control plane architecture and the corresponding signaling requirements. As the future telecommunication services will result from a convergence between B-ISDN and IN this paper takes into account the current progress of B-ISDN Capability Set 3 (B-ISDN CS3) standardization as well as the work done for IN Capability Set 2 (IN-CS2), and also the work done IN and Mobile integration (ETSI CAMEL).

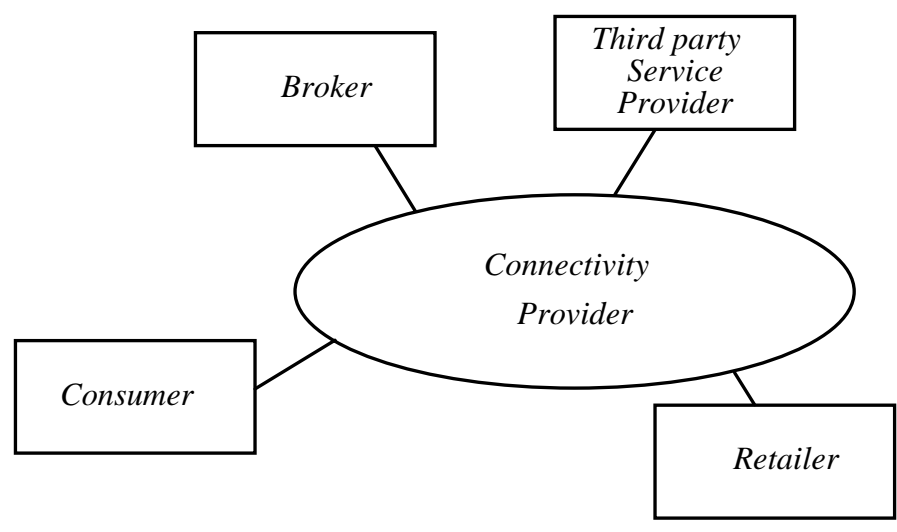

Fig. 1 : Business roles in the B-ISDN network 
We propose a control architecture compatible with these standards. However, our approach has a more generic scope in order to allow for the full range of future services requirements. It will, then, be shown how the proposed control architecture leads to a functional model providing a proper analysis of the required signaling capabilities and signaling network architecture and performance. Some examples of new services will be shown.

\section{2) A business model for B-ISDN}

As pointed out by the TINA-C work, future broadband telecommunication, will involve a different business structure than the present organization of narrowband communication. As it is already the case in today's mobile network, the consumer will generally seek to fulfill his telecommunication needs through an access provider or retailer type of enterprise. By means of this access provider he will reach services of all kinds, many of them provided by third party service suppliers, found through broker services. The classical telecommunication operating company business role will be considered more as a connectivity provider role rather than the global role it has today.

\section{3) B-ISDN CS3 and the Service / Connection separation: Session and Call}

Before B-ISDN CS3, the concept of a call was equivalent to the negotiation and allocation of communication resources between users, independently of service specific functionalities, and user roles in the service. Such a call concept was mixing in the same process the logical establishment of the association between parties, the search for network resources and the actual connection of the bearer facilities between these resources and parties.

New telecommunication services associated with the broadband and mobile networks cannot be satisfied by such a monolithic call approach for many reasons. Among them, we should notice that :

- some future services may not require any connection at all. For example, an alarm service may be done simply in the control plane with no user plane connection.

- some future services on the contrary may require several connections, eventually in different networks.

- terminal-related communications will be replaced by person-oriented communications, keeping track of the user profile, on whatever terminal he is using.

- services requiring precious resources like large bandwidth will require an efficient use of network resources. This will require to provide the possibility, before the seizure of any user plane bandwidth, of checking the state of the terminal, the subscription status of the called party for the peculiar service, the acceptance of the service by the person using the terminal.

- future services will require the negotiation of the traffic features (Quality of Service, bandwidth), during all the phases of the call (establishment and active phase)

- future services will require multimedia, multiconnection type of communication with possible configuration changes during a call. 
- future services involving complex call processing features would be more flexibly handled and the network would be less burdened if the complexity is not repeated link by link at every node involved in the call.

To overcome the difficulty of dealing with so many different networks requirements depending on the services, B-ISDN CS3 creates two different concepts : The Service Session concept and the Call concept, this last one being now considerable changed from it's former meaning.

The Service Session in B-ISDN CS3 is a single instance of service use with global significance. It includes the end to end view between user applications, while the call includes the end to end view between network access points. The Service Session takes place in the service provider platform (eventually distributed over several servers linked by networks). The Service Session takes care of service parameters like coding schemes, but does not take care of network aspects like QoS parameters.

On the other hand the call takes place in the network (or the networks) involved by the service. In it's new meaning, the call represents an association of one or more parties, using a telecommunication service to communicate through the network. The correspondence between session and calls may be of the "one to many" type, since a given Service Session may involve several calls or no calls at all.

A session may be kept set up, while the corresponding call is released for some time. Upon resumption of the session activity a new call will be set up.

In this new definition, the call has an end to end significance and does not include the establishment and release of the actual link by link bearer connections.

We can therefore state that the Service/Connection separation is actually a double separation: The Service / Call separation on one hand and the Call / Bearer separation on the other hand.

Due to the Call / Bearer separation, the old concept of a call is thus replaced with three communication functions organized in a layered manner.

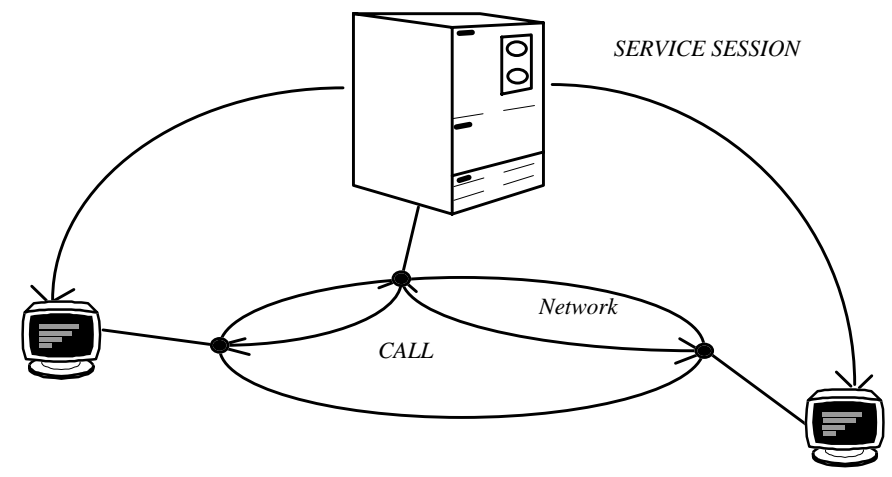

Fig. 2 : Service Session and calls in the B-ISDN network

The Call Control Function (CCF), in its new definition, is in charge of maintaining a connectivity tree between all the participating parties, and of maintaining an accepted quality 
of service over each connection within the network. It requires the services of the resource allocation function, itself requiring the services of the bearer connection function.

The Resource Control Function (RCF) optimizes the network usage by searching and reserving the most adequate network resources. These resources might be conference bridges, multicast points, protocol converters, intelligent peripherals such as voice or videoservers, or any special device required for a given service.

The Bearer Control Function (BCF) is then activated to create Bearer Connections between all the ports determined by the Resource control. New bearer connections may be added to a call or deleted from a call without the need of releasing or repeating the call and each new connection may be established with it's own routing.

\section{4) The need for an Access Session}

Due to mobility, it will be very frequent that the user of a given terminal will not be it's owner. However each user will have unique characteristics such as environment preferences, personal subscriptions and so on.

It is therefore advisable, following again the TINA approach, to introduce, in the clientserver manner, a separation between pure service logic aspects on one hand, and customer, location, and terminal dependent aspects on the other hand. This separation is provided by the Access Session, which provides the mean of selecting the service, localizing which terminal the involved parties are using, of verifying their subscriptions, of maintaining the user's preferences. More generally, it takes care of customer related functionalities, like the Home Location Register (HLR) of mobile telephony does it today, and presents a generic interface to the Service Session.

The Access Session might be executed on an operating company platform, or eventually on the platform of some retailer business acting as an intermediate between wholesale service providers and connectivity providers.

\section{5) A control architecture}

All above considerations finally lead to the control plane functional architecture shown in Figure 3. This functional architecture is complemented in Figure 4 by a physical architecture necessary to describe the signaling protocols and the physical establishment of the signaling links. This architecture entail the following functional entities.

- Terminal Manager Function (TMF) : terminal related, provided by the operating company supplying connection services to the terminal in use. The TMF is a generalization of the role played by the Visitor's Location Register (VLR) of mobile telephone networks. It keeps informations on the consumers using its related terminals.

- Access Session Function (ASF) : customer related, provided by a retailer type of business which may also be an operating company. The ASF is a generalization of the role played by the Home Location Register (HLR) of mobile telephone networks. It keeps informations on the exact status of all consumers associated with this access provider.

- Service Session Control Function (SSCF) : third party service supplier related or operating company related. The switches will normally contain the SSCF for basic services 
- Call Control Function (CCF) : B-ISDN switch related

- Resource Control Function (RCF) : B-ISDN switch related

- Bearer Control (BCF) : B-ISDN switch related

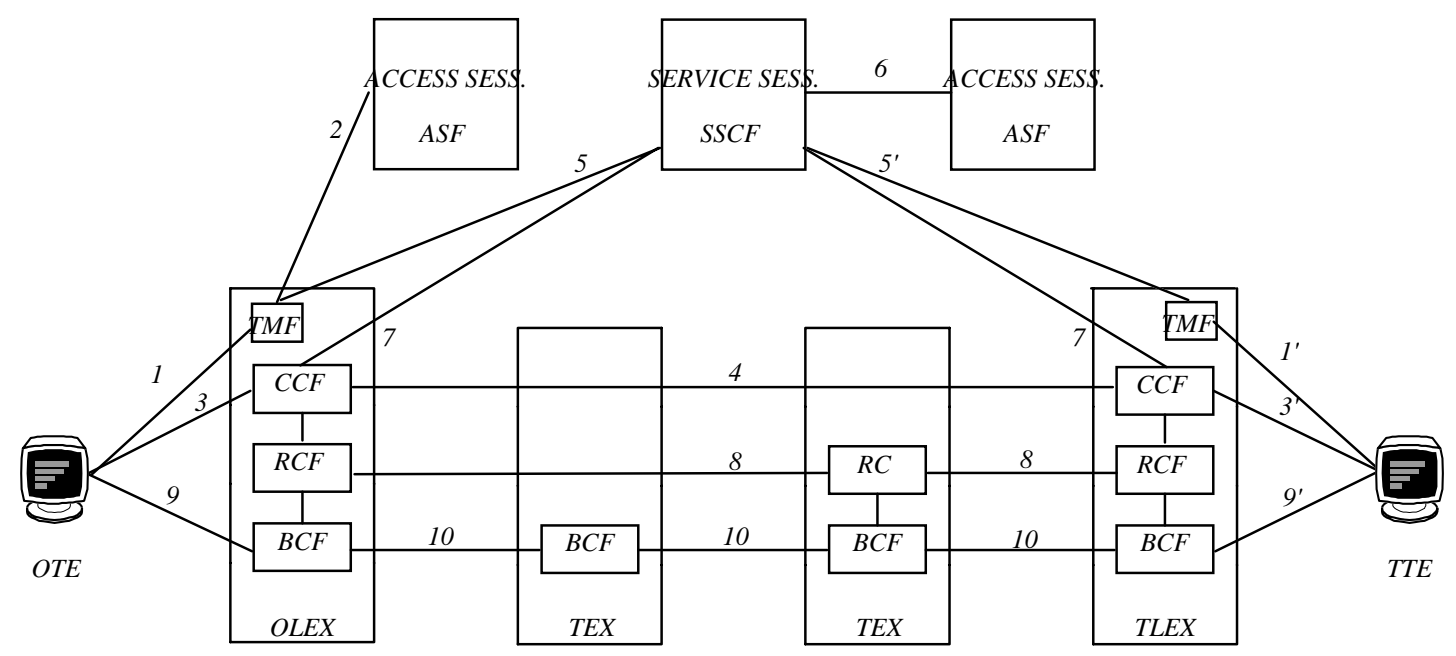

Fig . 3 : Control plane Functional Architecture

\section{6) The Service Session Precedence Principle}

The problem now resides in the sequencing and interactions of these functions. In IN CS-1 and IN CS-2, the call model is used as a device to trigger a service instance, giving therefore precedence of the call over the Service Session. The main issue then, is of the feasibility of an "universal, any service call model". Could any type of service be triggered from a call ? This idea is very much challenged and some services are known today as "call unrelated" services (such as updating the location of a mobile user, a short message service, etc.).

It seems then, that the key point of the control architecture lies in activating the Service Session prior to any call attempt by means of some direct signaling between the consumer and the Service Session. If required, the call or the calls (some services may require several calls in different networks) may then be originated, either by an instruction of the service instance to the originating customer equipment, or as a direct third party connection request to the network.

Our thesis is, that only in this manner, the service sequencing can be designed independently from the call sequences, and an universal call model, liberated from the task of service triggering, may be designed.

To back this statement we can point out that the Service Capability Sets constitute an open set. Therefore, if the services are call triggered, new services may require modifications of the call model. As an example, the Basic Call State Model (BCSM) had to be modified between IN CS1 and IN CS2. On the contrary, the precedence of the service over the call closes the set of functions required from calls. A generic call mechanism may then be designed. 
The originality of our architecture then, in respect to the currently proposed B-ISDN CS3 architecture, lies in the systematic precedence given to the service instanciation over the call instanciation. The mean for this systematic precedence is given by the provision of a direct signaling link between the Consumer and the Access Session Platform, as well as the Access Session platform and the Service provider platform. Our architecture satisfies the service goals of IN CS2 by the provision of a call independent mechanism for service triggering, and by a clear identification of the access function. Our architecture obeys the service objectives put forward by TINA-C. It differs, however, from the TINA-C architecture by the means of it's implementation, based on signaling. Our proposed architecture is thus a candidate for unifying all these various approaches to future broadband and multimedia communication.

\section{7) Some definitions}

In order to describe how a service session is activated and calls are set up in our architecture, we must first define the concepts of "user profile", "login file", and explain a principle that we consider fundamental to the architecture, namely the "Economy Principle".

\section{User Profile}

We call "user profile" a set of informations characterizing a particular consumer that are necessary to be known in order to establish telecommunication services for this consumer. Examples of such informations are :

- his prefered graphic environment (including wallpaper, colors, screen saver...)

- pointers for his various mail services (email, voice mails...)

- his bookmarks

- his applications

- his billing records

- his subscriptions in the case of non generaly available services

- his list of terminals associated to his eventual "one number" service. (a list of terminals where he is likely to be joined)

It is the responsability of the retailer or access provider to keep the user profile available and updated in the Access Session Function. A copy of this user profile is sent to the Terminal Manager Function of the switch hosting the terminal that is currently used by this consumer.

\section{Login file}

We call "login file" an information element sent by means of signaling by the terminal to Terminal Manager Function which contains among others :

- the identity of the calling consumer (unrelated to the terminal)

- the identity of the retailer or access session provider for this consumer

This Login file may be memorized on various media, such as a SIM card or any other portable memory device, that would be generally accepted by all terminal equipments.

\section{The Economy Principle}

We shall use consistently this principle according to which no signaling process should be made systematic if not strictly required. As an example, if the user profile is already cached in 
the Terminal Manager Function (like it will be in most cases), no signaling should take place to the Access Session Function.

\section{8) A Physical Architecture}

We describe now the mapping of the functional entities over physical machines.

The Terminal Manager Point (TMP) is a platform executing the Terminal Manager Function, tightly linked to an operating company's B-ISDN switch. This machine should be located at the operating company premises for safety and availability reasons and because of it's role in the billing operations.

The Access Session Point (ASP) is a platform executing the Access Session Function. It is a part of the Retailer (Access Provider) equipment and is located at his premises.

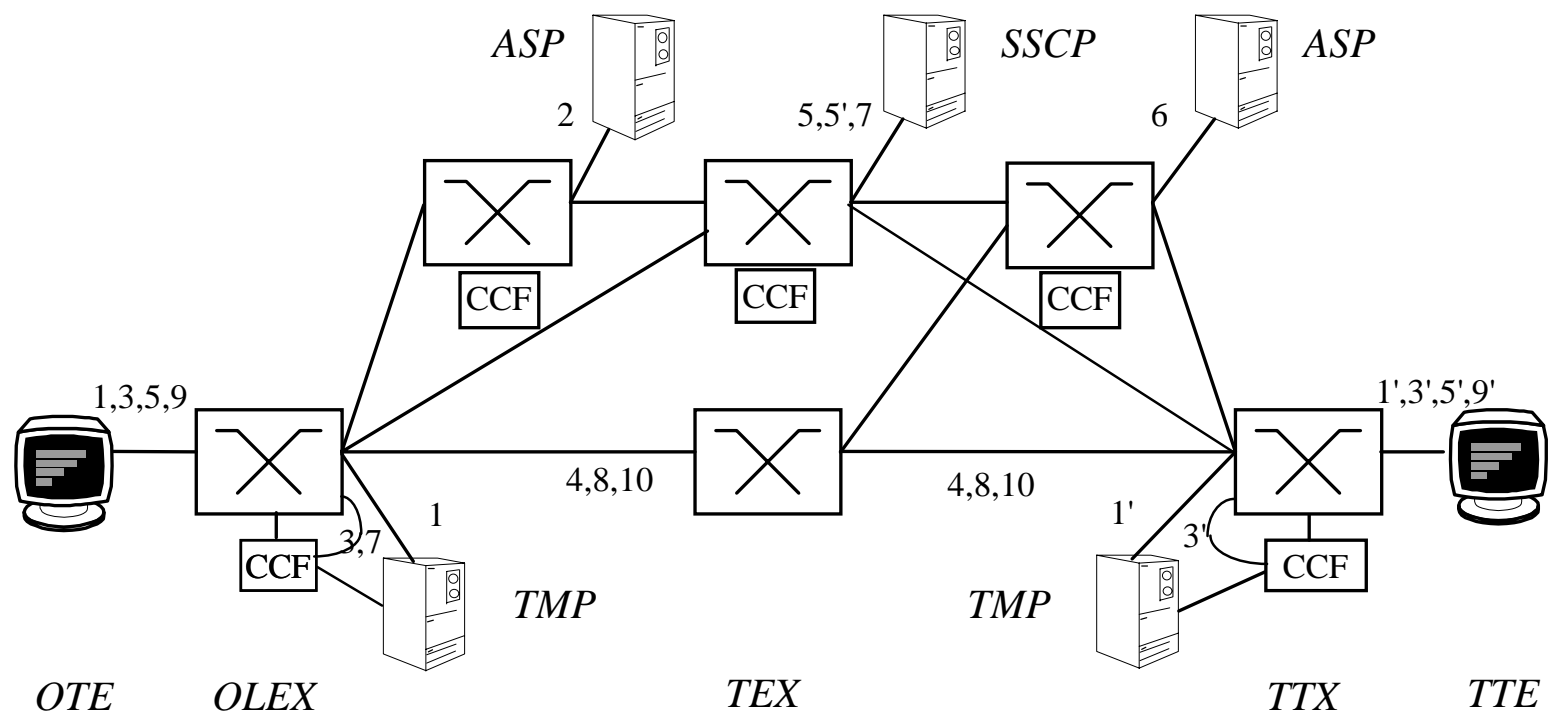

Fig . 4 : Control plane Physical Architecture

The Service Session Control Point (SSCP) is a platform executing the Service Session Function. It is a part of the service provider equipment and is located at his premises.

\section{9) A Signaling scheme for B-ISDN and IN integration}

According to Figures 3 and 4, a user logs in from an Originating Terminal Equipment (OTE) and the login file is sent by the OTE to a Terminal Manager Point (TMP) at the Originating Local Exchange (OLEX) location (signaling path 1). The login file contains, among others, the identification of the consumer presently using the terminal and the identity of the Access Service supplier for this user. If this user's profile is already cached in the TMF because he is already registered at this location, no signaling towards the Access Session Function is made according to the economy principle. However, if the user is new to this 
location, a signaling association between the TMF and the Access Session Function in the Access Session Point is established (signaling path 2). The ASP may be located anywhere, including in foreign countries. Through this signaling the TMF gets the user's profile containing this user's communication environment and receives updates of his communication services like written or voice mail pointers. He may then select a service, either from his bookmarks or after consultation of a broker service. When only a basic service is required (like a simple telephone call) the SSCF will be normally located right there, in the switch. For more sophisticated services, the service provider platform is reached (signaling path 5), access operations like subscription verification and authentication are performed between the TMF and the Service Session and user interaction with the Service Session may proceed. If other parties are involved in the service (such as a video server or an other person), a broker service may be invoked to find out the location of the retailer in charge of the terminating party and a new Access Session instance is created at this retailer's platform (signaling path 6). This access session determines the subscription status of this new party and its current location due to possible mobility. Some screen pop or any other pertinent indication is sent to this party to request his acceptance of this peculiar service instance (signaling paths 5', 1'). If acceptation takes place and if the service requires network connections, a call has to be originated in the network. It can be done in two different ways. In a first method the terminating parties addresses are loaded into the user's OTE and the call is originated via signaling from the OTE (signaling path 3). In an alternate method, the call is originated as a third party connection request sent by the service session to the call control function of the OLEX (signaling path 7). The call may now proceed by a Call Control instance, including look ahead procedure to know if the terminals are able to accept the call and to negotiate the QoS (signaling paths 4, 3 ), resource control is activated to locate suitable network resources (signaling path 8), and bearer control is finally performed to set up all the bearer connections (signaling paths $\left.9,10,9^{\prime}\right)$.

\section{0) Characteristics of the Signaling paths and protocols}

The signaling paths that we have described between all participating functional entities, have to satisfy different operational requirements that we describe briefly.

The functions of Signaling Path 1 are similar to the procedures of the Mobility Management (MM) protocol (location, authentication...). A connectionless mode of operation between the OTE and the TMF seems adequate for this path.

Signaling paths 2 and 6 are similar to the signaling path between a VLR and an HLR. The exchange of Data has a transactional character and the protocol appears to be an enrichment of the Mobile Application Part (MAP) of mobile networks. It will require the services of a full network layer similar to the Signaling Connection Control Part (SCCP) of Signaling System $\mathrm{n}^{\circ} 7$ (SS7), used in a connectionless mode and with an extended address translation capability. It would also require the services of a Remote Operation Service Element such as the Transaction Capability Application Part (TCAP) of SS7.

Signaling paths 3 and 9 are of the User to Network Interface (UNI) type. Some variations of the present Q2931 signaling protocols may be used at this level for Call Control Setup (path 3) and Bearer Control Setup (path 9). Here again connectionless modes of operation between the OTE and the $\mathrm{CCF}$ and BCF may be adequate for these paths. 
Signaling path 4 is the Call Control Signaling with the look ahead function. It is a new type of non circuit associated signaling protocol. It will also be based on such services as SCCP used in a connectionless mode and where the extended address translation capability is fundamental for the look ahead capability. It would also be based on such services as TCAP.

Signaling path 5 is the User Interaction with the Service Provider. It is a new type of non circuit associated signaling protocol, based on such services as SCCP and TCAP.

Signaling path 7 is a third party call request done directly by a Service Session, without the participation of the originating consumer or of his OTE. It is a new type of non circuit associated signaling protocol, having some similarities to the IN Intelligent Network Application Part (INAP).

Signaling path 8 is the Resource Control Signaling. It is also a new type of non circuit associated signaling protocol requiring the services of SCCP used in a connectionless mode and of TCAP. Special Real Time applications for locating and reserving network resources have to be developed here.

Signaling Path 10 is of the Network to Network Interface type (NNI) for Bearer connections. It is a circuit associated type of signaling of the type currently being defined as B-ISUP (Q2761).

\section{1) Service examples}

We now analyze the contents of the signaling information flows in the case of simple services.

\section{One Number Service}

This service allows a consumer to be reached on a number of terminals, some of them maybe in different networks. For example a call is addressed to the called party workstation and also to his GSM phone and also to his Internet phone service : In one possible implementation, the call might be presented simultaneously to all the terminals of a pre-declared list of networks and terminals. This list is kept updated in the ASF of the called party. When setting up the service the SSCF, probably located for such a simple service in the OLEX consults the ASF of the called party (signaling path 6) and get the list of terminals to contact. By means of signaling paths 7, 4 and 3', setup signals get sent to all the involved terminals. If one of the terminals sends back a connect message, all the other setup messages are canceled. Therefore no connections are made until an effective answer takes place.

\section{Videoconferencing with simultaneous translation}

Such a service may be supplied by a third party service supplier who owns special conference bridges equipment and who maintains a staff of persons qualified as translators and all having a common language. These persons usually work from their homes. Let's say that consumers $\mathrm{A}, \mathrm{B}$ and $\mathrm{C}$, all of them talking different languages decide to have a videoconference together. A simple way to do this service would be to establish by means of signaling paths $5,6,5$ ' a session between Consumers A, B, C, and three translators A', B', C', each one knowing in addition to the common language the language of his associated consumer. Once the session is setup a bidirectional videocall has to be made between $\mathrm{A}, \mathrm{B}, \mathrm{C}$, with a simple monodirectional 
broadcast to $\mathrm{A}^{\prime}, \mathrm{B}^{\prime}, \mathrm{C}$ since the translators do not produce images. For Voice a 3-port conference has to be established between $\mathrm{A}^{\prime}, \mathrm{B}^{\prime}, \mathrm{C}^{\prime}$ and three ordinary 2-port telephone calls A-A', B-B', C-C'. In order to achieve the proper connections, the Service Session gives third party connection orders to the call control functions of the Local exchanges of A, B, C, A', B', $\mathrm{C}^{\prime}$ by means of signaling path and protocol 7 . A total of 12 connections is setup according to Figure 5.

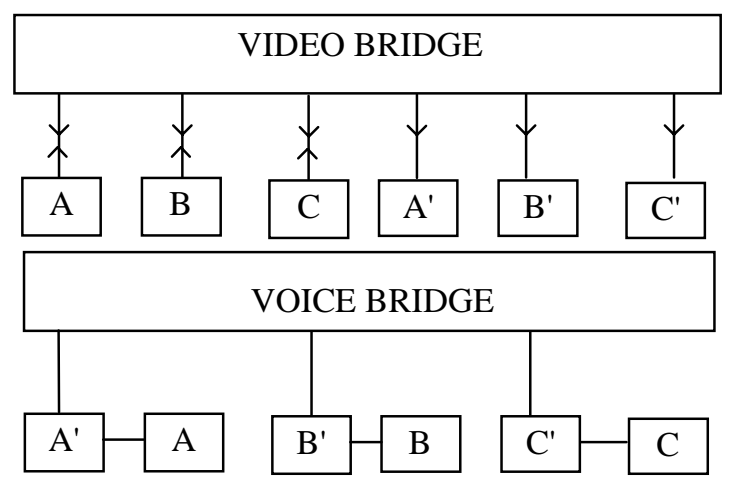

Fig . 5 : Connection topology for a Videoconference with simultaneous translation

\section{2) Conclusion}

We may conclude from this paper, that the difficulties of triggering services through a call may be solved by the introduction of direct signaling to an Access Session prior to any call establishment. In this manner mobility aspects may be taken in account and a clear separation between a Service Session that is not involved with the network aspects and calls that take care exclusively of these aspects is provided. In this manner the call functions may be defined as generic network functions for whatever type of telecommunication service. The architecture derived from this proposal clearly identifies the information flows required for the different types of services and therefore shows possible directions for subsequent studies on the B-ISDN network control plane. 


\section{References}

[1] B-ISDN UNI specification, ITU-T Recommendation Q2931.

[2] B-ISDN ITU-T Recommendations Q2761 to Q2764.

[3] B-ISDN CS3, ITU-T Recommendations Signaling requirements. Feb 96

[4] IN CS2, ITU-T Recommendations Q 1221 to Q 1229

[5] Emmanuel Darmois

TINA: from concept to reality.

Proceedings ICIN 1996, Bordeaux

[6] W.J. Barr, T. Boyd, Y.Inoue

The TINA Initiative

IEEE Communications Magazine, March 1993

[7] MAP ETS 300 599, ETSI Recommendation.February 1995

[8] TS/SMG-010278QR, ETSI Recommendation : CAMEL Service Definition

[9] RACE MAGIC R2044 :Multiservice Applications Governing Integrated Control

[10] A. Paglialunga, M. Siviero

Signaling protocol evolution : from narrowband to target broadband ISDN

IEEE Communications Magazine, 1992

[11] A. Paglialunga, M. Siviero

ISCP, the best candidate for the Target B-ISDN protocol

Communications Networks Vol. 4 n² (Mar-Apr 1993)

[12] A. Paglialunga, L. Ronchetti, L. Faglia, S. Minzer

The control of network ressources for complex B-ISDN services.

Proceedings of IFIP TC6 Broadband's Communication's 94, Paris

[13] T. Kovacikova, C. Rigault, S. Tohmé

Signaling evolution for B-ISDN.

Proceedings of Telkom 95, Midrand, South Africa. 